\title{
SOME GRONWALL-BELLMAN TYPE INEQUALITIES ON TIME SCALES FOR VOLTERRA-FREDHOLM DYNAMIC INTEGRAL EQUATIONS
}

\author{
A. A. El-Deeb \\ Department of Mathematics, Faculty of Science, Al-Azhar University, \\ Nasr City (11884), Cairo, Egypt. \\ Email ahmedeldeeb@azhar.edu.eg
}

Received 11/11/2016

Revised 26/3/2017

Accepted 13/8/2017

\begin{abstract}
In this paper, we prove several new explicit estimations for the solutions of some classes of nonlinear dynamic inequalities of Gronwall-Bellman-Pachpatte type on time scales. Our results formulate some integral and discrete inequalities discussed in the literature as special cases and extend some known dynamic inequalities on time scales. The inequalities given here can be used in the analysis of the qualitative properties of certain classes of dynamic equations on time scales. Some examples are presented to demonstrate the applications of our results.
\end{abstract}

MSC: 26D10; 26D15; 26D20; 34A12; 34A40

keywords:dynamic inequalities, Gronwall-Bellman type, time scales calculus

\section{Introduction}

In various situations, we are interested in knowing qualitative properties of solutions without explicit knowledge of the solution process. One of the best known and widely used inequalities in the study of qualitative properties of solutions of nonlinear differential equations can be stated as follows:

Theorem 1.1. Let $u$ be a continuous function defined on the interval $D=[\alpha, \alpha+h]$ and

$$
0 \leq u(t) \leq \int_{\alpha}^{t}[\delta u(s)+\gamma] d s
$$

for all $t \in D$, where $\alpha, \gamma, \delta$ and $h$ are nonnegative constants. Then

$$
0 \leq u(t) \leq \gamma h e^{\delta h} .
$$

The inequality given in Theorem 1.1, was discovered by Thomas Gronwall [1] in 1919. In the recent years, these inequalities have been greatly enriched by the recognition of their potential and intrinsic worth in many applications of the applied sciences, (see [2-12]). In 1943, Richard Bellman in [13], proved the fundamental inequality (see Theorem 1.2) named Gronwall-Bellman's inequality as a generalization for Gronwall's inequality and plays a very important role in studying stability and asymptotic behaviour of solutions of linear differential-difference equations.

Theorem 1.2. Let $u$ and $f$ be continuous and nonnegative functions defined on $[\alpha, \beta]$, and let $c$ be nonnegative constant. Then the inequality

$$
u(t) \leq c+\int_{\alpha}^{t} f(s) u(s) d s, \quad t \in[\alpha, \beta],
$$

implies that

$$
u(t) \leq c \exp \left(\int_{\alpha}^{t} f(s) d s\right), \quad t \in[\alpha, \beta] .
$$

Bellman in [14] proved and made use of the following variant of the inequality given by himself in Theorem 1.2. 
Theorem 1.3. Let $u$ and $f$ be continuous and nonnegative functions defined on $[\alpha, \beta]$, and let a be a continuous, positive and nondecreasing function defined on $[\alpha, \beta]$; then

$$
u(t) \leq a(t)+\int_{\alpha}^{t} f(s) u(s) d s, \quad t \in[\alpha, \beta],
$$

implies that

$$
u(t) \leq a(t) \exp \left(\int_{\alpha}^{t} f(s) d s\right), \quad t \in[\alpha, \beta] .
$$

Gollwitzer [15] gave the following generalization of the Gronwall-Bellman inequality:

$$
u(t) \leq f(t)+g(t) \int_{\alpha}^{t} h(s) u(s) d s, \quad t \in[\alpha, \beta] .
$$

A fairly general version of Theorem 1.2 is given in the following theorem by Pachpatte [16]:

$$
u(t) \leq p(t)+q(t) \int_{\alpha}^{t}[f(s) u(s)+g(s)] d s, \quad t \in[\alpha, \beta] .
$$

In [17], Pachpatte established also the following inequality:

$$
w(t) \leq c(t)+\int_{a}^{t} f(s) w(s) d s+\int_{a}^{b} g(s) w(s) d s,
$$

for all $t \in[a, b] \subseteq \mathbb{R}$. Kender et al. [18] established the following further generalizations of the inequality (1.5) proved by of Pachpatte in [17] where he replaced the linear term of the unknown function $\omega$ by nonlinear term $\omega^{p}$ in both sides of the inequality as following

$$
w^{p}(t) \leq c(t)+\int_{a}^{t} f(s) w(s) d s+\int_{a}^{b} g(s) w^{p}(s) d s,
$$

for all $t \in[a, b] \subseteq \mathbb{R}$. It is well known that, the dynamic inequalities play an important role in the development of the qualitative theory of dynamic equations on time scales. The study of dynamic equations on time scales which goes back to Stefan Hilger [19] becomes an area of mathematics and recently has received a lot of attention. The general idea is to prove a result for a dynamic equation or a dynamic inequality where the domain of the unknown function is a so called time scale $\mathbb{T}$, which may be an arbitrary closed subset of the real numbers $\mathbb{R}$ see $[20,21]$. The purpose of the theory of time scales is to unify continuous and discrete analysis. The three most popular examples of calculus on time scales are differential calculus, difference calculus, and quantum calculus (see [22]), i.e, when $\mathbb{T}=\mathbb{R}, \mathbb{T}=\mathbb{N}$ and $\mathbb{T}=q^{N_{0}}=\left\{q^{t}: t \in N_{0}\right\}$ where $q>1$. The book on the subject of time scales by Bohner and Peterson [23] summarizes and organizes much of time scale calculus. During the past decade a number of dynamic inequalities has been established by some authors which are motivated by some applications, for example, we refer the reader to [23-26] for contributions, and the references cited therein.

In this paper, we present some new nonlinear dynamic inequalities on an arbitrarily time scale $\mathbb{T}$, these dynamic inequalities unify and extend the inequalities presented in [17] and [18]. Our main results will be proved by employing some useful inequalities which will be presented in Section 2. The paper is organized as in the following: In Section 2, some basic concepts of the calculus on time scales and useful lemmas are introduced. In Section 3, we state and prove the main results. In Section 4 , we present several applications to study some qualitative properties of the solutions of certain dynamic equations.

\section{Basic Results and Lemmas on Time Scales}

In this section, we present some background on time scales. A time scale $\mathbb{T}$ is an arbitrary nonempty closed subset of the real numbers. The time scales calculus was initiated by Hilger in his $\mathrm{PhD}$ thesis in order to unify discrete and continuous analysis [19]. We assume throughout that $\mathbb{T}$ has the topology that it inherits from the standard topology on the real numbers $\mathbb{R}$. For $t \in \mathbb{T}$, first we define the forward jump operator $\sigma: \mathbb{T} \rightarrow \mathbb{T}$ by:

$$
\sigma(t):=\inf \{s \in \mathbb{T}: s>t\},
$$


and second, the backward jump operator $\rho: \mathbb{T}: \rightarrow \mathbb{T}$ by:

$$
\rho(t):=\sup \{s \in \mathbb{T}: s<t\} .
$$

In this definition, we put $\inf \emptyset=\sup \mathbb{T}$ and $\sup \emptyset=\inf \mathbb{T}$, where $\emptyset$ is the empty set. A point $t \in \mathbb{T}$ with $\inf \mathbb{T}<t<\sup \mathbb{T}$, is said to be left-dense if $\rho(t)=t$ and is right-dense if $\sigma(t)=t$, points that are simultaneously right-dense and left-dense are said to be dense, is left-scattered if $\rho(t)<t$ and right-scattered if $\sigma(t)>t$, points that are simultaneously right-scattered and left-scattered are said to be isolated. A function $g: \mathbb{T} \rightarrow \mathbb{R}$ is said to be right-dense continuous (rd-continuous) provided $g$ is continuous at right-dense points and at left-dense points in $\mathbb{T}$, left-sided limits exist and are finite. The set of all such rd-continuous functions is denoted by $C_{r d}(\mathbb{T})$. A function $f: \mathbb{T} \rightarrow \mathbb{R}$ is said to be left-dense continuous (ld-continuous) provided $f$ is continuous at left-dense points and at right-dense points in $\mathbb{T}$, right-sided limits exist and are finite. The set of all such ld-continuous functions is denoted by $C_{l d}(\mathbb{T})$.

The forward and backward graininess functions $\mu$ and $\nu$ for a time scale $\mathbb{T}$ is defined by $\mu(t):=\sigma(t)-t$, and $\nu(t):=t-\rho(t)$, respectively.

Given a time scale $\mathbb{T}$, we introduce the sets $\mathbb{T}^{\kappa}, \mathbb{T}_{\kappa}$, and $\mathbb{T}_{\kappa}^{\kappa}$ as follows. If $\mathbb{T}$ has a left-scattered maximum $t_{1}$, then $\mathbb{T}^{\kappa}=\mathbb{T}-\left\{t_{1}\right\}$, otherwise $\mathbb{T}^{\kappa}=\mathbb{T}$. If $\mathbb{T}$ has a right-scattered minimum $t_{2}$, then $\mathbb{T}^{\kappa}=\mathbb{T}-\left\{t_{2}\right\}$, otherwise $\mathbb{T}^{\kappa}=\mathbb{T}$. Finally, $\mathbb{T}_{\kappa}^{k}=\mathbb{T}^{\kappa} \cap \mathbb{T}_{\kappa}$.

The interval $[a, b]$ in $\mathbb{T}$ is defined by

$$
[a, b]_{\mathbb{T}}=\{\tau \in \mathbb{T}: a \leq \tau \leq b\} .
$$

Open intervals and half-closed interval are defined similarly.

Let $f: \mathbb{T} \rightarrow \mathbb{R}$ be a real-valued function on a time scale $\mathbb{T}$. Then for all $t \in \mathbb{T} \kappa$, we define $f^{\Delta}(t)$ to be the number (if it exists) with the property that given any $\varepsilon>0$ there is a neighborhood $U$ of $t$ such that

$$
\left|[f(\sigma(t))-f(s)]-f^{\Delta}(t)[\sigma(t)-s]\right| \leq \varepsilon|\sigma(t)-s|, \quad \forall s \in U .
$$

For $f: \mathbb{T} \rightarrow \mathbb{R}$, we define the function $f^{\sigma}: \mathbb{T} \rightarrow \mathbb{R}$ by $f^{\sigma}(t)=f(\sigma(t))$ for all $t \in \mathbb{T}$, that is, $f^{\sigma}=f \circ \sigma$. Similarly, we define the function $f^{\rho}: \mathbb{T} \rightarrow \mathbb{R}$ by $f^{\rho}(t)=f(\rho(t))$ for all $t \in \mathbb{T}$, that is, $f^{\rho}=f \circ \rho$. A time scale $\mathbb{T}$ is said to be regular if the following two conditions are satisfied simultaneously: (1) $\sigma(\rho(t))=t$ and (2) $\rho(\sigma(t))=t, \forall t \in \mathbb{T}$. The product and quotient rules for the derivative of the product $f g$ and the quotient $f / g$ (where $g g^{\sigma} \neq 0$, here $g^{\sigma}=g \circ \sigma$ ) of two differentiable functions $f$ and $g$ are given as the following:

$$
(f g)^{\Delta}(t)=f^{\Delta}(t) g(t)+f(\sigma(t)) g^{\Delta}(t)=f(t) g^{\Delta}(t)+f^{\Delta}(t) g(\sigma(t)),
$$

and

$$
\left(\frac{f}{g}\right)^{\Delta}(t)=\frac{f^{\Delta}(t) g(t)-f(t) g^{\Delta}(t)}{g(t) g(\sigma(t))} .
$$

A function $F: \mathbb{T} \rightarrow \mathbb{R}$ is called a delta antiderivative of $f: \mathbb{T} \rightarrow \mathbb{R}$ provided that $F^{\Delta}(t)=f(t)$ holds for all $t \in \mathbb{T}^{\kappa}$, and the delta integral of $f$ is defined by

$$
\int_{a}^{b} f(t) \Delta t=F(b)-F(a) .
$$

We will frequently use the following useful relations between calculus on time scales $\mathbb{T}$ and differential calculus on $\mathbb{R}$, difference calculus on $\mathbb{Z}$, and quantum calculus on $\overline{q^{\mathbb{Z}}}$. Note that

(i) if $\mathbb{T}=\mathbb{R}$, then

$$
\sigma(t)=t, \quad \mu(t)=0, \quad f^{\Delta}(t)=f^{\prime}(t), \quad \int_{a}^{b} f(t) \Delta t=\int_{a}^{b} f(t) d t
$$

(ii) if $\mathbb{T}=\mathbb{Z}$, then

$$
\sigma(t)=t+1, \quad \mu(t)=1, \quad f^{\Delta}(t)=\Delta f(t), \quad \int_{a}^{b} f(t) \Delta t=\sum_{t=a}^{b-1} f(t) ;
$$

(iii) and if $\mathbb{T}=\overline{q^{\mathbb{Z}}}=\left\{q^{k}: k \in \mathbb{Z}\right\} \cup\{0\}, q>1$, then

$$
\sigma(t)=q t, \quad \mu(t)=(q-1) t, \quad \int_{a}^{b} f(t) \Delta t=(q-1) \sum_{k=\log _{q}(a)}^{\log _{q}(b)-1} q^{k} f\left(q^{k}\right), \quad \forall a, b \in q^{\mathbb{N}_{0}} .
$$


It can be shown (see [23]) that if $g \in C_{r d}(\mathbb{T})$, then the Cauchy integral $G(t):=\int_{t_{0}}^{t} g(s) \Delta s$ exists, $t_{0} \in \mathbb{T}$, and satisfies $G^{\Delta}(t)=g(t), t \in \mathbb{T}$. An infinite integral is defined as

$$
\int_{a}^{\infty} f(t) \Delta t=\lim _{b \rightarrow \infty} \int_{a}^{b} f(t) \Delta t
$$

Now, we will give the definition of the generalized exponential function and its derivatives. We say that $p: \mathbb{T} \rightarrow \mathbb{R}$ is regressive provided $1+\mu(t) p(t) \neq 0$ for all $t \in \mathbb{T}^{\kappa}$, we define the set $\Re$ of all regressive and rd-continuous functions. We define the set $\Re^{+}$of all positively regressive elements of $\Re$ by $\Re^{+}=\{p \in \Re: 1+\mu(t) p(t)>0, \forall t \in \mathbb{T}\}$. The set of all regressive functions on a time scale $\mathbb{T}$ forms an Abelian group under the addition $\oplus$ defined by $p \oplus q=p+q+\mu p q$. If $p \in \Re$, then we can define the exponential function by

$$
e_{p}(t, s)=\exp \left(\int_{s}^{t} \xi_{\mu(\tau)}(p(\tau)) \Delta \tau\right), \quad s, t \in \mathbb{T},
$$

where $\xi_{h}(z)$ is the cylinder transformation, which is defined by

$$
\xi_{h}(z)= \begin{cases}\frac{\log (1+h z)}{h}, & h \neq 0 \\ z, & h=0\end{cases}
$$

If $p \in \Re$, then $e_{p}(t, s)$ is real-valued and nonzero on $\mathbb{T}$. If $p \in \Re^{+}$, then $e_{p}\left(t, t_{0}\right)$ is always positive.

Note that

- if $\mathbb{T}=\mathbb{R}$, then

$$
e_{a}\left(t, t_{0}\right)=\exp \left(\int_{t_{0}}^{t} a(s) d s\right)
$$

- if $\mathbb{T}=\mathbb{Z}$, then

$$
e_{a}\left(t, t_{0}\right)=\prod_{s=t_{0}}^{t-1}(1+a(s))
$$

- if $\mathbb{T}=q^{\mathbb{N}_{0}}$, then

$$
e_{a}\left(t, t_{0}\right)=\prod_{s=t_{0}}^{t-1}(1+(q-1) s a(s)) .
$$

In the following, we present the basic lemmas that will be needed in the proof of our main results.

Lemma 2.1 ( [27]). If $p, q \in \Re$ and $a, b, c \in \mathbb{T}$, then

1. $e_{p}(t, t)=1$ and $e_{0}(t, s)=1$;

2. $e_{p}(\sigma(t), s)=(1+\mu(t) p(t)) e_{p}(t, s)$;

3. if $p \in \Re^{+}$, then $e_{p}\left(t, t_{0}\right)>0, \forall t \in \mathbb{T}$;

4. $\int_{a}^{b} p(t) e_{p}(c, \sigma(t)) \Delta t=-\int_{a}^{b}\left[e_{p}(c, \cdot)\right]^{\Delta}(t) \Delta t=e_{p}(c, a)-e_{p}(c, b)$.

Lemma 2.2 (See [27]). If $p \in \Re$ and fix $t \in \mathbb{T}$, then the exponential function $e_{p}\left(t, t_{0}\right)$ is the unique solution of the following initial value problem:

$$
\left\{\begin{array}{l}
y^{\Delta}(t)=p(t) y(t) \\
y\left(t_{0}\right)=1
\end{array}\right.
$$


Lemma 2.3 (See [27]). Let $t_{0} \in \mathbb{T}^{\kappa}$ and $k: \mathbb{T} \times \mathbb{T}^{\kappa} \rightarrow \mathbb{R}$ be continuous at $(t, t)$, where $t>t_{0}$ and $t \in \mathbb{T}^{\kappa}$. Assume that $k^{\Delta}(t, \cdot)$ is rd-continuous on $\left[t_{0}, \sigma(t)\right]$. If for any $\varepsilon>0$, there exists a neighborhood $U$ of $t$, independent of $\tau \in\left[t_{0}, \sigma(t)\right]$, such that

$$
\left|[k(\sigma(t), \tau)-k(s, \tau)]-k^{\Delta}(t, \tau)[\sigma(t)-s]\right| \leq \varepsilon|\sigma(t)-s|, \quad \forall s \in U .
$$

If $k^{\Delta}$ denotes the derivative of $k$ with respect to the first variable, then

$$
f(t)=\int_{t_{0}}^{t} k(t, \tau) \Delta \tau
$$

yields

$$
f^{\Delta}(t)=\int_{t_{0}}^{t} k^{\Delta}(t, \tau) \Delta \tau+k(\sigma(t), t) .
$$

Lemma 2.4 ( [27]). Suppose $u, b \in C_{r d}$ and $a \in \Re^{+}$. Then

$$
u^{\Delta}(t) \leq a(t) u(t)+b(t), \quad t \geq t_{0}, t \in \mathbb{T}^{\kappa}
$$

yields

$$
u(t) \leq u\left(t_{0}\right) e_{a}\left(t, t_{0}\right)+\int_{t_{0}}^{t} e_{a}(t, \sigma(\tau)) b(\tau) \Delta \tau, \quad t \geq t_{0}, t \in \mathbb{T}^{\kappa} .
$$

Lemma 2.5 ( [28]). If $x \geq 0$ and $p \geq 1$, then

$$
x^{\frac{1}{p}} \leq m_{1} x+m_{2},
$$

where $m_{1}=\frac{1}{p} K^{\frac{1-p}{p}}, m_{2}=\frac{p-1}{p} K^{\frac{1}{p}}$ and $K>0$.

Now we are ready to state and prove our main results, which give us the time scales version of the inequalities proved in [17] and [18].

\section{Main results}

In this section, we will state and prove the main results and investigate some dynamic Gronwall-Bellman inequalities on time scales.

Theorem 3.1. Let $a, b \in \mathbb{T}_{k}^{k}$ with $a<b$ and $\omega, g, f, c \in C_{r d}\left([a, b]_{\mathbb{T}^{k}}, \mathbb{R}^{+}\right)$, c be delta-differentiable on $\mathbb{T}$ with $c^{\Delta}(t) \geq 0$, and $p \geq 1$ be a constant. If

$$
\omega^{p}(t) \leq c(t)+\int_{a}^{t} g(s) \omega(s) \Delta s+\int_{a}^{b} f(s) \omega^{p}(s) \Delta s
$$

for all $t \in[a, b]_{\mathbb{T}^{k}}$, then

$$
\omega(t) \leq\left\{M e_{m_{1} g}(t, a)+\int_{a}^{t} e_{m_{1} g}(t, \sigma(s)) A(s) \Delta s\right\}^{\frac{1}{p}}
$$

where

$$
A(t)=c^{\Delta}(t)+m_{2} g(t),
$$

and

$$
M=\frac{c(a)+\int_{a}^{b} f(s)\left[\int_{a}^{s} e_{m_{1} g}(s, \sigma(\lambda)) A(\lambda) \Delta \lambda\right] \Delta s}{1-\int_{a}^{b} f(s) e_{m_{1} g}(s, a) \Delta s},
$$

such that

$$
\int_{a}^{b} f(s) e_{m_{1} g}(s, a) \Delta s<1,
$$

where $m_{1}, m_{2}$ are defined as in Lemma 2.5, and $e_{m_{1} g}(t, a)$ is a solution of the initial value problem (2.9) in Lemma 2.2 when $p(t)$ replaced by $m_{1} g$. 
Proof. Define a function $z_{1}(t)$ by

$$
z_{1}(t)=c(t)+\int_{a}^{t} g(s) \omega(s) \Delta s+\int_{a}^{b} f(s) \omega^{p}(s) \Delta s,
$$

then, we get that

$$
\omega(t) \leq z_{1}^{\frac{1}{p}}(t)
$$

and

$$
z_{1}(a)=c(a)+\int_{a}^{b} f(s) \omega^{p}(s) \Delta s,
$$

from (3.6), and using (3.7), we have:

$$
z_{1}^{\Delta}(t)=c^{\Delta}(t)+g(t) \omega(t) \leq c^{\Delta}(t)+g(t) z_{1}^{\frac{1}{p}}(t) .
$$

Therefore, using Lemma 2.5, from (3.9), we get that

$$
\begin{aligned}
z_{1}^{\Delta}(t) & \leq c^{\Delta}(t)+m_{1} g(t) z_{1}(t)+m_{2} g(t) \\
& =m_{1} g(t) z_{1}(t)+\left[c^{\Delta}(t)+m_{2} g(t)\right] \\
& =m_{1} g(t) z_{1}(t)+A(t)
\end{aligned}
$$

where $A(t)$ is as defined in (4.6). Now an application of Lemma 2.3 to (3.10) yields

$$
z_{1}(t) \leq z_{1}(a) e_{m_{1} g}(t, a)+\int_{a}^{t} e_{m_{1} g}(t, \sigma(s)) A(s) \Delta s,
$$

from (3.7) and (3.11), we get that

$$
\omega^{p}(t) \leq z_{1}(a) e_{m_{1} g}(t, a)+\int_{a}^{t} e_{m_{1} g}(t, \sigma(s)) A(s) \Delta s,
$$

from (3.8) and (3.12), we have

$$
\begin{aligned}
z_{1}(a)= & c(a)+\int_{a}^{b} f(s) \omega^{p}(s) \Delta s \\
\leq & c(a)+\int_{a}^{b} f(s)\left[z_{1}(a) e_{m_{1} g}(s, a)+\int_{a}^{s} e_{m_{1} g}(s, \sigma(\lambda)) A(\lambda) \Delta \lambda\right] \Delta s \\
\leq & c(a)+\int_{a}^{b} f(s) z_{1}(a) e_{m_{1} g}(s, a) \Delta s \\
& +\int_{a}^{b} f(s)\left[\int_{a}^{s} e_{m_{1} g}(s, \sigma(\lambda)) A(\lambda) \Delta \lambda\right] \Delta s .
\end{aligned}
$$

Thus from (3.13), we obtain

$$
z_{1}(a) \leq \frac{c(a)+\int_{a}^{b} f(s)\left[\int_{a}^{s} e_{m_{1} g}(s, \sigma(\lambda)) A(\lambda) \Delta \lambda\right] \Delta s}{1-\int_{a}^{b} f(s) e_{m_{1} g}(s, a) \Delta s}=M,
$$

and then we get the required inequality (3.2) from (3.12) and (3.14). The proof is complete.

Remark 3.1. If we put $p=1$, and $\mathbb{T}=\mathbb{R}$, in Theorem 3.1 and using relation (2.3), then we get the continuous inequality result due to Pachpatte in [17]. 
Remark 3.2. By taking $\mathbb{T}=\mathbb{R}$ in Theorem 3.1 and using the relation (2.3), it is easy to observe that the inequality obtained in Theorem 3.1 reduces to the inequality obtained by Kender et al. in [18, Theorem 2.1].

As a special case of Theorem 3.1, if $\mathbb{T}=\mathbb{Z}$ and using the relations (2.4) and (2.7), we obtain the following discrete result.

Corollary 3.1. Let $\mathbb{T}=\mathbb{Z}$ and assume that $\omega, g, c$ and $f$ are nonnegative sequences defined for $t \in \mathbb{N}_{0}$, then the inequality

$$
\omega^{p}(t) \leq c(t)+\sum_{s=a}^{t-1} g(s) \omega(s)+\sum_{s=a}^{b-1} f(s) \omega^{p}(s)
$$

implies,

$$
\omega(t) \leq\left\{\tilde{M} \prod_{s=a}^{t-1}\left(1+m_{1} g(s)\right)+\sum_{s=a}^{t-1} \hat{A}(s) \prod_{\tau=s+1}^{t-1}\left(1+m_{1} g(\tau)\right)\right\}^{\frac{1}{p}}
$$

where

$$
\hat{A}(t)=c(t+1)-c(t)+m_{2} g(t),
$$

and,

$$
\hat{M}=\frac{c(a)+\sum_{s=a}^{b-1} f(s)\left[\sum_{\lambda=a}^{s-1} \hat{A}(\lambda) \prod_{\tau=\lambda+1}^{s-1}\left(1+m_{1} g(\tau)\right)\right]}{1-\sum_{s=a}^{b-1} f(s) \prod_{\tau=\lambda}^{s-1}\left(1+m_{1} g(\tau)\right)},
$$

where,

$$
\sum_{s=a}^{b-1} f(s) \prod_{\tau=a}^{s-1}\left(1+m_{1} g(\tau)\right)<1
$$

Theorem 3.2. Let $a, b \in \mathbb{T}_{k}^{k}$ with $a<b$ and $\omega, g, f, c \in C_{r d}\left([a, b]_{\mathbb{T}^{k}}, \mathbb{R}^{+}\right)$, c be delta-differentiable on $\mathbb{T}$ with $c^{\Delta}(t) \geq 0$, and $k(t, s), k^{\Delta}(t, s) \in C_{r d}\left([a, b]_{\mathbb{T}^{k}} \times[a, b]_{\mathbb{T}^{k}}, \mathbb{R}^{+}\right)$for $a \leq s \leq t \leq b$ and $p \geq 1$ be a constant. If

$$
\omega^{p}(t) \leq c(t)+\int_{a}^{t} k(t, s) \omega(s) \Delta s+\int_{a}^{b} g(s) \omega^{p}(s) \Delta s,
$$

for all $t \in[a, b]_{\mathbb{T}^{k}}$, then

$$
\omega(t) \leq\left\{M_{1} e_{m_{1} \eta}(t, a)+\int_{a}^{t} A_{1}(s) e_{m_{1} \eta}(t, \sigma(s)) \Delta s\right\}^{\frac{1}{p}}
$$

where

$$
\begin{gathered}
A_{1}(t)=c^{\Delta}(t)+m_{2} \eta(t), \\
\eta(t)=k(\sigma(t), t)+\int_{a}^{t} k^{\Delta}(t, \tau) \Delta \tau,
\end{gathered}
$$

and

$$
M_{1}=\frac{c(a)+\int_{a}^{b} g(s)\left[\int_{a}^{s} e_{m_{1} \eta}(s, \sigma(\tau)) A_{1}(\tau) \Delta \tau\right] \Delta s}{1-\int_{a}^{b} g(s) e_{m_{1} \eta}(s, a) \Delta s},
$$

such that

$$
\int_{a}^{b} g(s) e_{m_{1} \eta}(s, a) \Delta s<1,
$$

where $m_{1}, m_{2}$ are defined as in Lemma 2.5, and $e_{m_{1} \eta}(t, a)$ is a solution of the initial value problem (2.9) in Lemma 2.2 when $p(t)$ replaced by $m_{1} \eta$.

Proof. Define a function $z_{2}(t)$ by

$$
z_{2}(t)=c(t)+\int_{a}^{t} k(t, s) \omega(s) \Delta s+\int_{a}^{b} g(s) \omega^{p}(s) \Delta s
$$


then, we get that

$$
\omega(t) \leq z_{2}^{\frac{1}{p}}(t)
$$

and

$$
z_{2}(a)=c(a)+\int_{a}^{b} g(s) \omega^{p}(s) \Delta s .
$$

Using Lemma 2.3 in (3.21) and from (3.22), we have

$$
\begin{aligned}
z_{2}^{\Delta}(t) & =c^{\Delta}(t)+k(\sigma(t), t) \omega(t)+\int_{a}^{t} k^{\Delta}(t, \tau) \omega(\tau) \Delta \tau \\
& \leq c^{\Delta}(t)+k(\sigma(t), t) z_{2}^{\frac{1}{p}}(t)+\int_{a}^{t} k^{\Delta}(t, \tau) z_{2}^{\frac{1}{p}}(\tau) \Delta \tau \\
& \leq c^{\Delta}(t)+\left[k(\sigma(t), t)+\int_{a}^{t} k^{\Delta}(t, \tau) \Delta \tau\right] z_{2}^{\frac{1}{p}}(t) \\
& =c^{\Delta}(t)+\eta(t) z_{2}^{\frac{1}{p}}(t),
\end{aligned}
$$

where $\eta(t)$ is define as in (3.18). Using Lemma 2.5 in (3.24), the inequality (3.24) can be written as,

$$
\begin{aligned}
z_{2}^{\Delta}(t) & \leq c^{\Delta}(t)+m_{1} \eta(t) z_{2}(t)+m_{2} \eta(t) \\
& =m_{1} \eta(t) z_{2}(t)+\left[c^{\Delta}(t)+m_{2} \eta(t)\right] \\
& =m_{1} \eta(t) z_{2}(t)+A_{1}(t)
\end{aligned}
$$

where $A_{1}(t)$ is define as in (3.17). Now, using Lemma 2.4 in (3.25) yields that

$$
z_{2}(t) \leq z_{2}(a) e_{m_{1} \eta}(t, a)+\int_{a}^{t} e_{m_{1} \eta}(t, \sigma(\tau)) A_{1}(s) \Delta \tau
$$

from (3.22) and (3.26), we get that

$$
\omega^{p}(t) \leq z_{2}(a) e_{m_{1} \eta}(t, a)+\int_{a}^{t} e_{m_{1} \eta}(t, \sigma(\tau)) A_{1}(\tau) \Delta \tau
$$

From (3.23) and (3.27), we have

$$
\begin{aligned}
z_{2}(a)= & c(a)+\int_{a}^{b} g(s) \omega^{p}(s) \Delta s \\
\leq & c(a)+\int_{a}^{b} g(s)\left[z_{2}(a) e_{m_{1} \eta}(s, a)+\int_{a}^{s} e_{m_{1} \eta}(s, \sigma(\tau)) A_{1}(\tau) \Delta \tau\right] \Delta s \\
\leq & c(a)+\int_{a}^{b} g(s) z_{2}(a) e_{m_{1} \eta}(s, a) \Delta s \\
& +\int_{a}^{b} g(s)\left[\int_{a}^{s} e_{m_{1} \eta}(s, \sigma(\tau)) A_{1}(\tau) \Delta \tau\right] \Delta s .
\end{aligned}
$$

Thus from (3.28), we obtain

$$
z_{2}(a) \leq \frac{c(a)+\int_{a}^{b} g(s)\left[\int_{a}^{s} e_{m_{1} \eta}(s, \sigma(\tau)) A_{1}(\tau) \Delta \tau\right] \Delta s}{1-\int_{a}^{b} g(s) e_{m_{1} \eta}(s, a) \Delta s}=M_{1}
$$

for all $t \in[a, b]_{\mathbb{T}^{k}}$, we get the required inequality (3.16) from (3.27) and (3.29). The proof is complete. 
Remark 3.3. By taking $\mathbb{T}=\mathbb{R}$ in Theorem 3.2 and using the relation (2.3), it is easy to observe that the inequality obtained in Theorem 3.2 reduces to the inequality obtained by Kender et al. in [18, Theorem 2.2].

As a special case of Theorem 3.2, if $\mathbb{T}=\mathbb{Z}$ and using the relations (2.4) and (2.7), we get the following discrete result.

Corollary 3.2. Let $\mathbb{T}=\mathbb{Z}$ and assume that $\omega, g, k(t, s), \Delta k(t, s), c$ and $f$ are nonnegative sequences defined for $t \in \mathbb{N}_{0}$, then the inequality

$$
\omega^{p}(t) \leq c(t)+\sum_{s=a}^{t-1} k(t, s) \omega(s)+\sum_{s=a}^{b-1} g(s) \omega^{p}(s)
$$

implies,

$$
\omega(t) \leq\left\{\hat{M}_{1} \prod_{s=a}^{t-1}\left(1+m_{1} \eta(s)\right)+\sum_{s=a}^{t-1} \hat{A}_{1}(s) \prod_{\tau=s+1}^{t-1}\left(1+m_{1} \eta(\tau)\right)\right\}^{\frac{1}{p}}
$$

where

$$
\hat{A}_{1}(t)=c(t+1)-c(t)+m_{2} \eta(t)
$$

and

$$
\begin{aligned}
\eta(t) & =k(\sigma(t), t)+\sum_{s=a}^{t-1} \Delta k(t, s), \\
& =k(t+1, t)+\sum_{s=a}^{t-1} \Delta k(t, s),
\end{aligned}
$$

where $\Delta k(t, s)=k(t+1, s)-k(t, s)$, and

$$
\hat{M}_{1}=\frac{c(a)+\sum_{s=a}^{b-1} g(s)\left[\sum_{\lambda=a}^{s-1} \hat{A}_{1}(\lambda) \prod_{\tau=\lambda+1}^{s-1}\left(1+m_{1} \eta(\tau)\right)\right]}{1-\sum_{s=a}^{b-1} g(s) \prod_{\tau=\lambda}^{s-1}\left(1+m_{1} \eta(\tau)\right)},
$$

where

$$
\sum_{s=a}^{b-1} g(s) \prod_{\tau=a}^{s-1}\left(1+m_{1} \eta(\tau)\right)<1
$$

Theorem 3.3. Let $\omega$ and $c$ be defined as in Theorem 3.2, $k_{1}(t, s), k_{2}(t, s), k_{1}^{\Delta}(t, s)$ and $k_{2}^{\Delta}(t, s) \in C_{r d}\left([a, b]_{\mathbb{T}^{k}} \times[a, b]_{\mathbb{T}^{k}}, \mathbb{R}^{+}\right)$ for $a \leq s \leq t \leq b$ and $p \geq 1$ be a constant. Assume that $a, b \in \mathbb{T}_{k}^{k}$ with $a<b$. If

$$
\omega^{p}(t) \leq c(t)+\int_{a}^{t} k_{1}(t, s) \omega(s) \Delta s+\int_{a}^{b} k_{2}(t, s) \omega^{p}(s) \Delta s
$$

for all $t \in[a, b]_{\mathbb{T}^{k}}$, then

$$
\omega(t) \leq\left\{M_{2} e_{m_{1} E_{1}+E_{2}}(t, a)+\int_{a}^{t} A_{2}(s) e_{m_{1} E_{1}+E_{2}}(t, \sigma(s)) \Delta s\right\}^{\frac{1}{p}}
$$

where

$$
M_{2}=\frac{c(a)+\int_{a}^{b} k_{2}(a, s)\left[\int_{a}^{s} A_{2}(\lambda) e_{m_{1} E_{1}+E_{2}}(s, \sigma(\lambda)) \Delta \lambda\right] \Delta s}{1-\int_{a}^{b} k_{2}(a . s) e_{m_{1} E+1+E_{2}}(s, a) \Delta s}
$$

such that

$$
\int_{a}^{b} k_{2}(s, a) e_{m_{1} E_{1}+E_{2}}(s, a) \Delta s<1
$$

where

$$
E_{1}(t)=\int_{a}^{t} k_{1}^{\Delta}(t, s) \Delta s+k_{1}(\sigma(t), t)
$$




$$
\begin{gathered}
E_{2}(t)=\int_{a}^{t} k_{2}^{\Delta}(t, s) \Delta s, \\
A_{2}(t)=c^{\Delta}(t)+m_{2} E_{1}(s),
\end{gathered}
$$

where $m_{1}, m_{2}$ are as defined in Lemma 2.5, and $e_{m_{1} E_{1}+E_{2}}(t, a)$ is a solution of the initial value problem (2.9) in Lemma 2.2, when $p(t)$ replaced by $m_{1} E_{1}+E_{2}$.

Proof. Define a function $z_{3}(t)$ by

$$
z_{3}(t)=c(t)+\int_{a}^{t} k_{1}(t, s) \omega(s) \Delta s+\int_{a}^{b} k_{2}(t, s) \omega^{p}(s) \Delta s,
$$

from (3.34) we have $z_{3}(t) \geq 0$ nondecreasing function with

$$
z_{3}(a)=c(a)+\int_{a}^{b} k_{2}(a, s) \omega^{p}(s) \Delta s
$$

and

$$
\omega(t) \leq z_{3}^{\frac{1}{p}}(t)
$$

from (3.34), and using (3.36), we have

$$
\begin{aligned}
z_{3}^{\Delta}(t)= & c^{\Delta}(t)+k_{1}(\sigma(t), t) \omega(t)+\int_{a}^{t} k_{1}^{\Delta}(t, s) \omega(s) \Delta s \\
& +\int_{a}^{b} k_{2}^{\Delta}(t, s) \omega^{p}(s) \Delta s \\
\leq & c^{\Delta}(t)+k_{1}(\sigma(t), t) z_{3}^{\frac{1}{p}}(t)+\int_{a}^{t} k_{1}^{\Delta}(t, s) z_{3}^{\frac{1}{p}}(s) \Delta s \\
& +\int_{a}^{b} k_{2}^{\Delta}(t, s) z_{3}(s) \Delta s \\
\leq & c^{\Delta}(t)+\left[k_{1}(\sigma(t), t)+\int_{a}^{t} k_{1}^{\Delta}(t, s) \Delta s\right] z_{3}^{\frac{1}{p}}(t) \\
& +\int_{a}^{b} k_{2}^{\Delta}(t, s) z_{3}(s) \Delta s \\
= & c^{\Delta}(t)+E_{1} z_{3}^{\frac{1}{p}}(t)+E_{2}(t) z_{3}(s) .
\end{aligned}
$$

By using Lemma 2.5 on $z_{3}^{\frac{1}{p}}(t)$ and $z_{3}(s)$ in (3.37), we have

$$
z_{3}^{\Delta}(t) \leq\left[m_{1} E_{1}(t)+E_{2}(t)\right] z_{3}(t)+c^{\Delta}(t)+m_{2} E_{1}(t) .
$$

Therefore, using Lemma (2.4) in (3.38) we get that

$$
z_{3}(t) \leq z_{3}(a) e_{m_{1} E_{1}+E_{2}}(t, a)+\int_{a}^{t}\left[c^{\Delta}(s)+m_{2} E_{1}(s)\right] e_{m_{1} E_{1}+E_{2}}(t, \sigma(s)) \Delta s,
$$

from (3.36) and (3.39), we get that

$$
\omega^{p}(t) \leq z_{3}(a) e_{m_{1} E 1+E 2}(t, a)+\int_{a}^{t} A_{2}(s) e_{m_{1} E_{1}+E_{2}}(t, \sigma(s)) \Delta s,
$$


from (3.35) and (3.40), we have

$$
\begin{aligned}
z_{3}(a) \leq & c(a)+\int_{a}^{b} k_{2}(a, s)\left[z_{3}(a) e_{m_{1} E_{1}+E_{2}}(s, a)\right. \\
& \left.+\int_{a}^{s} A_{2}(\lambda) e_{m_{1} E_{1}+E_{2}}(s, \sigma(\lambda)) \Delta \lambda\right] \Delta s \\
\leq & c(a)+\int_{a}^{b} k_{2}(a . s) z_{3}(a) e_{m_{1} E_{1}+E_{2}}(s, a) \Delta s \\
& +\int_{a}^{b} k_{2}(a, s) \int_{a}^{s} A_{2}(\lambda) e_{m_{1} E_{1}+E_{2}}(s . \sigma(\lambda)) \Delta \lambda \Delta s
\end{aligned}
$$

Thus from (3.41), we obtain

$$
z_{3}(a) \leq \frac{c(a)+\int_{a}^{b} k_{2}(a, s)\left[\int_{a}^{s} A_{2}(\lambda) e_{m_{1} E_{1}+E_{2}}(s, \sigma(\lambda)) \Delta \lambda\right] \Delta s}{1-\int_{a}^{b} k_{2}(a, s) e_{m_{1} E_{1}+E_{2}}(s, a) \Delta s}=M_{2}
$$

and then we get the required inequality (3.31) from (3.40) and (3.42). The proof is complete.

Remark 3.4. By taking $\mathbb{T}=\mathbb{R}$ in Theorem 3.3 and using the relation (2.3), it is easy to observe that the inequality obtained in Theorem 3.3 reduces to the inequality obtained by Kender et al. in [18, Theorem 2.3].

As a special case of Theorem 3.3, if $\mathbb{T}=\mathbb{Z}$ and using the relations (2.4) and (2.7), we obtain the following discrete result.

Corollary 3.3. Let $\mathbb{T}=\mathbb{Z}$ and assume that $\omega, k_{1}(t, s), k_{2}(t, s)_{2}, \Delta k_{1}(t, s), \Delta k_{2}(t, s), c$ and $f$ are nonnegative sequence defined for $t \in \mathbb{N}_{0}$, then the inequality

$$
\omega^{p}(t) \leq c(t)+\sum_{s=a}^{t-1} k_{1}(t, s) \omega(s)+\sum_{s=a}^{b-1} k_{2}(t, s) \omega^{p}(s)
$$

implies,

$$
\omega(t) \leq\left\{\hat{M}_{2} \prod_{s=a}^{t-1}\left(1+m_{1} E_{1}(s)+E_{2}(s)\right)+\sum_{s=a}^{t-1} \hat{A}_{2}(s) \prod_{\tau=s+1}^{t-1}\left(1+m_{1} E_{1}(\tau)+E_{2}(\tau)\right)\right\}^{\frac{1}{p}}
$$

where

$$
\hat{A}_{2}(t)=c(t+1)-c(t)+m_{2} E_{1}(t),
$$

and

$$
E_{1}(t)=k_{1}(t+1, t)+\sum_{s=a}^{t-1} \Delta k_{1}(t, s)
$$

where $\Delta k_{1}(t, s)=k_{1}(t+1, s)-k_{1}(t, s)$, and

$$
E_{2}(t)=\sum_{s=a}^{t-1} \Delta k_{2}(t, s)
$$

where $\Delta k_{2}(t, s)=k_{2}(t+1, s)-k_{2}(t, s)$, and

$$
\hat{M}_{2}=\frac{c(a)+\sum_{s=a}^{b-1} k_{2}(a, s)\left[\sum_{a=\lambda}^{s-1} \hat{A}_{2}(\lambda) \prod_{\tau=\lambda+1}^{s-1}\left(1+m_{1} E_{1}(\tau)+E_{2}(\tau)\right)\right]}{1-\sum_{s=a}^{b-1} k_{2}(a, s) \prod_{\tau=\lambda}^{s-1}\left(1+m_{1} E_{1}(\tau)+E_{2}(\tau)\right)}
$$

where,

$$
\sum_{s=a}^{b-1} k_{2}(a, s)(s) \prod_{\tau=a}^{s-1}\left(1+m_{1} E_{1}(\tau)+E_{2}(\tau)\right)<1
$$


Theorem 3.4. Let $\omega$ and $c$ be defined as in Theorem 3.2, $k_{1}(t, s), k_{2}(t, s), k_{3}(t, s), k_{1}^{\Delta}(t, s), k_{2}^{\Delta}(t, s)$ and $k_{3}^{\Delta}(t, s) \in$ $C_{r d}\left([a, b]_{\mathbb{T}^{k}} \times[a, b]_{\mathbb{T}^{k}}, \mathbb{R}^{+}\right)$, and $p \geq 1$ be a constant. If $a, b \in \mathbb{T}_{k}^{k}$ and $a<b$, then

$$
\omega^{p}(t) \leq c(t)+\int_{a}^{t} k_{1}(t, s)\left[\omega(s)+\int_{a}^{s} k_{2}(s, \tau) \omega(\tau) \Delta \tau\right] \Delta s+\int_{a}^{b} k_{3}(t, s) \omega^{p}(s) \Delta s
$$

for all $t \in[a, b]_{\mathbb{T}^{k}}$, implies

$$
\omega(t) \leq\left\{M_{3} e_{m_{1} E_{3}+E_{4}}(t, a)+\int_{a}^{t} A_{3}(s) e_{m_{1} E 3+E 4}(t, \sigma(s)) \Delta s\right\}^{\frac{1}{p}}
$$

where

$$
M_{3}=\frac{c(a)+\int_{a}^{b} k_{3}(a, s)\left[\int_{a}^{s} A_{3}(\lambda) e_{m_{1} E_{3}+E_{4}}(s, \sigma(\lambda)) \Delta \lambda\right] \Delta s}{1-\int_{a}^{b} k_{3}(a, s) e_{m_{1} E_{3}+E_{4}}(s, a) \Delta s}
$$

such that

$$
\int_{a}^{b} k_{3}(s, a) e_{m_{1} E_{3}+E_{4}}(s, a) \Delta s<1
$$

where

$$
\begin{gathered}
E_{3}(t)=\quad \int_{a}^{t} k_{1}^{\Delta}(t, s)\left[1+\int_{a}^{s} k_{2}(s, \tau) \tau\right] \Delta s \\
+k_{2}(\sigma(t), t)\left[1+\int_{a}^{t} k_{2}(t, \tau) \Delta \tau\right], \\
E_{4}(t)=\int_{a}^{b} k_{3}^{\Delta}(t, s) \Delta s, \\
A_{3}(t)=c^{\Delta}(t)+m_{2} E_{3}(t),
\end{gathered}
$$

where $m_{1}, m_{2}$ are as defined in Lemma 2.5, and $e_{m_{1} E_{3}+E_{4}}(t, a)$ is a solution of the initial value problem (2.9) in Lemma 2.2, when $p(t)$ replaced by $m_{1} E_{3}+E_{4}$.

Proof. The proof is similar to the proof of Theorem 3.3.

Remark 3.5. By taking $\mathbb{T}=\mathbb{R}$ in Theorem 3.4 and using the relation (2.3), it is easy to observe that the inequality obtained in Theorem 3.3 reduces to the inequality obtained by Kender et al. in [18, Theorem 2.4].

As a special case of Theorem 3.4, if $\mathbb{T}=\mathbb{Z}$ and using the relations (2.4) and (2.7), we obtain the following discrete result.

Corollary 3.4. Let $\mathbb{T}=\mathbb{Z}$ and assume that $\omega, k_{1}(t, s), k_{2}(t, s)_{2}, \Delta k_{1}(t, s), \Delta k_{2}(t, s), c$ and $f$ are nonnegative sequence defined for $t \in \mathbb{N}_{0}$, then the inequality

$$
\omega^{p}(t) \leq c(t)+\sum_{s=a}^{t-1} k_{1}(t, s)\left[\omega(s)+\sum_{s=a}^{b-1} k_{2}(t, s) \omega(s)\right]+\sum_{s=a}^{b-1} k_{3}(t, s) \omega^{p}(s),
$$

implies

$$
\omega(t) \leq\left\{\hat{M}_{3} \prod_{s=a}^{t-1}\left(1+m_{1} E_{3}(s)+E_{4}(s)\right)+\sum_{s=a}^{t-1} \hat{A}_{3}(s) \prod_{\tau=s+1}^{t-1}\left(1+m_{1} E_{3}(\tau)+E_{4}(\tau)\right)\right\}^{\frac{1}{p}}
$$

where

$$
\hat{A}_{3}(t)=c(t+1)-c(t)+m_{2} E_{3}(t)
$$

and

$$
E_{3}(t)=\sum_{s=a}^{t-1} \Delta k_{1}(t, s)\left[1+\sum_{\tau=a}^{s-1} k_{2}(s, \tau)\right]+k_{2}(t+1, t)+\sum_{s=a}^{t-1} k_{1}(t, s)\left[1+\sum_{\tau=a}^{s-1} k_{2}(s, \tau)\right]
$$


where $\Delta k_{1}(t, s)=k_{1}(t+1, s)-k_{1}(t, s)$, and

$$
E_{4}(t)=\sum_{s=a}^{t-1} \Delta k_{3}(t, s)
$$

where $\Delta k_{2}(t, s)=k_{2}(t+1, s)-k_{2}(t, s)$, and

$$
\hat{M}_{3}=\frac{c(a)+\sum_{s=a}^{b-1} k_{3}(a, s)\left[\sum_{a=\lambda}^{s-1} \hat{A}_{3}(\lambda) \prod_{\tau=\lambda+1}^{s-1}\left(1+m_{1} E_{3}(\tau)+E_{4}(\tau)\right)\right]}{1-\sum_{s=a}^{b-1} k_{3}(a, s) \prod_{\tau=\lambda}^{s-1}\left(1+m_{1} E_{3}(\tau)+E_{4}(\tau)\right)},
$$

where

$$
\sum_{s=a}^{b-1} k_{3}(a, s)(s) \prod_{\tau=a}^{s-1}\left(1+m_{1} E_{3}(\tau)+E_{4}(\tau)\right)<1
$$

\section{Applications}

In this section, we present some applications of Theorem 3.1 and Theorem 3.3 to obtain the explicit estimates on the solutions of certain dynamic equations, and also prove the uniqueness and global existence of solutions for a class of nonlinear dynamic integral equations.

Consider the following dynamic integral equation on time scale

$$
\left(\omega^{p}\right)^{\Delta}(t)=l+F\left(s, \omega(s), \int_{a}^{b} H\left(t, s, \omega^{p}(s)\right) \Delta s\right), \quad \omega^{p}(a)=r,
$$

where $F: \mathbb{T}_{k}^{k} \times \mathbb{R}^{+} \times \mathbb{R}^{+} \rightarrow \mathbb{R}^{+}$is a continuous function, and $H: \mathbb{T}_{k}^{k} \times \mathbb{T}_{k}^{k} \times \mathbb{R}^{+} \rightarrow \mathbb{R}^{+}$is also a continuous function. Assume that

$$
\begin{gathered}
|r+l(t-s)| \leq c(t), \\
|F(s, \omega, \nu)| \leq|g(s) \omega(s)|+\int_{a}^{b}|\nu(s)| \Delta s, \\
|H(t, s, \omega)| \leq \frac{f(s) \omega^{p}(s)}{t-a}, \quad t \neq a,
\end{gathered}
$$

where $c, f, g \in C_{r d}\left([a, b]_{\mathbb{T}^{k}}, \mathbb{R}^{+}\right)$and $r, l$ are given constants.

Theorem 4.1. Consider the dynamical system (4.1), and suppose that we have the hypothesis (4.2), (4.3) and (4.4), then each solution $\omega$ of the dynamical system (4.1) under discussion verifies the following estimation

$$
\omega(t) \leq\left\{M e_{m_{1} g}(t, a)+\int_{a}^{t} e_{m_{1} g}(t, \sigma(s)) A(s) \Delta s\right\}^{\frac{1}{p}},
$$

for all $t \in[a, b]_{\mathbb{T}^{k}}$, where $M, A$ are defined as in the following:

$$
A(t)=c^{\Delta}(t)+m_{2} g(t),
$$

and

$$
M=\frac{c(a)+\int_{a}^{b} f(s)\left[\int_{a}^{s} e_{m_{1} g}(s, \sigma(\lambda)) A(\lambda) \Delta \lambda\right] \Delta s}{1-\int_{a}^{b} f(s) e_{m_{1} g}(s, a) \Delta s},
$$

such that

$$
\int_{a}^{b} f(s) e_{m_{1} g}(s, a) \Delta s<1
$$

where $m_{1}, m_{2}$ are defined as in Lemma 2.5. 
Proof. Let $\omega$ be a solution of the dynamical system (4.1). Then, we see that $\omega$ satisfies the following equivalent nonlinear dynamic integral equation

$$
\omega^{p}(t)=r+l(t-a)+\int_{a}^{t} F\left(s, \omega(s), \int_{a}^{b} H\left(\tau, \omega^{p}(s)\right) \Delta \tau\right) \Delta s
$$

from (4.9) and using the hypothesis (4.2), (4.3) and (4.4), we get that

$$
\begin{aligned}
\left.\mid \omega^{p}(t)\right) \mid & \leq|r+l(t-a)|+\int_{a}^{t}\left|F\left(s, \omega(s), \int_{a}^{b} H\left(\tau, s, \omega^{p}(s)\right) \Delta \tau\right)\right| \Delta s \\
& \leq|r+l(t-a)|+\int_{a}^{t}\left(g(s) \omega(s)+\int_{a}^{b} H\left(\tau, s, \omega^{p}(s)\right) \Delta \tau\right) \Delta s \\
& \leq c(t)+\int_{a}^{t} g(s) \omega(s) \Delta s+(t-a) \int_{a}^{b} H\left(\tau, s, \omega^{p}(\tau)\right) \Delta \tau \\
& \leq c(t)+\int_{a}^{t} g(s) \omega(s) \Delta s+\int_{a}^{b} f(s) \omega^{p}(s) \Delta s .
\end{aligned}
$$

Now applying Theorem 3.1, to (4.10), yields

$$
|\omega(t)| \leq\left\{M e_{m_{1} g}(t, a)+\int_{a}^{t} e_{m_{1} g}(t, \sigma(s)) A(s) \Delta s\right\}^{\frac{1}{p}} .
$$

This estimation is the required in (4.5). The proof is complete.

Example 4.1. Consider the following nonlinear dynamic integral equation in time scale

$$
\left.\omega^{p}(t)\right)=h(t)+F_{1}\left(t, \int_{a}^{t} H_{1}\left(s, \omega(s), k_{1}\right) \Delta s, \int_{a}^{b} H_{2}\left(s, \omega^{p}(s), k_{2}\right) \Delta s\right), \omega^{p}(a)=\tilde{r},
$$

if,

$$
\begin{aligned}
|h(t)| & \leq c(t), \\
\left|F_{1}(t, u, \tilde{\nu})\right| & \leq|u|+|\tilde{\nu}|, \\
\left|H_{1}\right| & \leq k_{1}(t, s) \omega(s), \\
\left|H_{2}\right| & \leq k_{2}(t, s) \omega(s),
\end{aligned}
$$

where $\omega, g, f, c \in C_{r d}\left([a, b]_{\mathbb{T}^{k}}, \mathbb{R}^{+}\right), c$ is delta-differentiable on $\mathbb{T}^{k}$ and $c^{\Delta}(t) \geq 0$, and $k_{1}(t, s), k_{1}^{\Delta}(t, s), k_{2}(t, s), k_{2}^{\Delta}(t, s)$ $\in C_{r d}\left([a, b]_{\mathbb{T}^{k}} \times[a, b]_{\mathbb{T}^{k}}, \mathbb{R}^{+}\right)$for $a \leq s \leq t \leq b, \tilde{r}$ and $p \geq 1$, then we have the explicit bound estimation of the solution $\omega$ of (4.11) as the following:

$$
\omega(t) \leq\left\{M_{2} e_{m_{1} E_{1}+E_{2}}(t, a)+\int_{a}^{t} e_{m_{1} E_{1}+E_{2}}(t, \sigma(s)) A_{2}(s) \Delta s\right\}^{\frac{1}{p}}
$$

for all $t \in[a, b]_{\mathbb{T}^{k}}$, where $M_{2}, A_{2}, E_{1}, E_{2}$ and $e_{m_{1} E_{1}+E_{2}}$ are defined as the following:

$$
M_{2}=\frac{c(a)+\int_{a}^{b} k_{2}(a, s)\left[\int_{a}^{s} A_{2}(\lambda) e_{m_{1} E_{1}+E_{2}}(s, \sigma(\lambda)) \Delta \lambda\right] \Delta s}{1-\int_{a}^{b} k_{2}(a . s) e_{m_{1} E+1+E_{2}}(s, a) \Delta s}
$$

such that

$$
\begin{gathered}
\int_{a}^{b} k_{2}(s, a) e_{m_{1} E_{1}+E_{2}}(s, a) \Delta s<1 \\
E_{1}(t)=\int_{a}^{t} k_{1}^{\Delta}(t, s) \Delta s+k_{1}(\sigma(t), t)
\end{gathered}
$$




$$
\begin{gathered}
E_{2}(t)=\int_{a}^{t} k_{2}^{\Delta}(t, s) \Delta s, \\
A_{2}(t)=c^{\Delta}(t)+m_{2} E_{1}(s),
\end{gathered}
$$

where $m_{1}, m_{2}$ are as defined in Lemma 2.5 .

Proof. From (4.11), (4.12), we have

$$
|\omega(t)|^{p} \leq c(t)+\int_{a}^{t} k_{1}(t, s)|\omega(s)| \Delta s+\int_{a}^{b} k_{2}(t, s)|\omega(s)|^{p} \Delta s .
$$

Now applying Theorem 3.3, to (4.16), we get

$$
\omega(t) \leq\left\{M_{2} e_{m_{1} E_{1}+E_{2}}(t, a)+\int_{a}^{t} e_{m_{1} E_{1}+E_{2}}(t, \sigma(s)) A_{2}(s) \Delta s\right\}^{\frac{1}{p}} .
$$

This is the desired estimation in (4.13). The proof is complete.

Example 4.2. Assume that

$$
\begin{aligned}
\left|F\left(t, \gamma_{1}, \gamma_{2}\right)-F_{1}\left(t, \beta_{1}, \beta_{2}\right)\right| & \leq\left|\gamma_{1}-\beta_{1}\right|+\left|\gamma_{2}-\beta_{2}\right| \\
\left|H_{1}\left(t, \omega_{1}\right)-H_{1}\left(t, \omega_{2}\right)\right| & \leq k_{1}(t, s)\left|\omega_{1}(s)-\omega_{2}(s)\right| \\
\left|H_{2}\left(t, \omega_{1}\right)-H_{2}\left(t, \omega_{2}\right)\right| & \leq k_{2}(t, s)\left|\omega_{1}^{p}(s)-\omega_{2}^{p}(s)\right| \\
\left|\omega_{1}(t)-\omega_{2}(t)\right| & \leq\left|\omega_{1}^{p}(t)-\omega_{2}^{p}(t)\right|,
\end{aligned}
$$

where $\omega(t), k_{1}(t, s)$ and $k_{2}(t, s), p$ are defined as in Example 4.1, then the dynamic equation (4.11) has at most one solution. Proof. Let $\omega_{1}$ and $\omega_{2}$ be two solutions of (4.11). Then we get that

$$
\begin{aligned}
\omega_{1}^{p}(s)-\omega_{2}^{p}(s)= & F\left(t, \int_{a}^{t} H_{1}\left(s, \omega_{1}(s)\right), \int_{a}^{b} H_{2}\left(s, \omega_{1}^{p}(s)\right) \Delta s\right) \\
& -F\left(t, \int_{a}^{t} H_{1}\left(s, \omega_{2}(s)\right), \int_{a}^{b} H_{2}\left(s, \omega_{2}^{p}(s)\right) \Delta s\right),
\end{aligned}
$$

from (4.17) and (4.18), we get that

$$
\left|\omega_{1}^{p}(s)-\omega_{2}^{p}(s)\right| \leq \int_{a}^{t} k_{1}(t, s)\left|\omega_{1}^{p}(s)-\omega_{2}^{p}(s)\right| \Delta s+\int_{a}^{b} k_{2}(t, s)\left|\omega_{1}^{p}(s)-\omega_{2}^{p}(s)\right| \Delta s .
$$

By Theorem 3.3, we have $\omega_{1}^{p}(s)-\omega_{2}^{p}(s)=0$. Therefor $\omega_{1}=\omega_{2}$. Then (4.11) has at most one solution. The proof is complete.

\section{Acknowledgment}

The author thanks the referees very much for their careful comments and valuable suggestions on this paper. 


\section{References}

[1] T. H. Gronwall. Note on the derivative with respect to a parameter of the solutions of a system of differential equations. Ann. of Math, 20 (1919):292-296.

[2] A. Abdeldaim and A. A. El-Deeb. On some generalizations of certain retarded nonlinear integral inequalities with iterated integrals and an application in retarded differential equation. J. Egy. Math. Soc., (23 (2015):470-475.

[3] A. Abdeldaim and A. A. El-Deeb. some new retarded nonlinear integral inequalities with iterated integrals and their application in differential-integral equation. JFCA, 5(3s)(2014)(9):1-9, 2014.

[4] A. Abdeldaim and A. A. El-Deeb. On generalized of certain retarded nonlinear integral inequalities and its applications in retarded integro-differential equations. Appl. Math. Comput., 256(2015):375-380.

[5] A. Abdeldaim and A. A. El-Deeb. On some new nonlinear retarded inte- gral inequalities with iterated integrals and their applications in integro- differential equations. Br. J. Math. Comp. Sc., 5(4)(2015):479-491.

[6] A. A. El-Deeb and Reda Gamal Ahmed. On some explicit bounds on certain retarded nonlinear integral inequalities with applications. Adv. Ineq. Appl., (2016) 17 pages.

[7] H. El-Owaidy, A. Abdeldaim, and A. A. El-Deeb. On some new retarded nonlinear integral inequalities and their applications. Math. Sci. Lett., 3, No. 3 (2014):157-164.

[8] S. S. Dragomir. Some Gronwall Type Inequalities and Applications. RGMIA Monographs, Vectoria University Australia, (2000) Online.

[9] B. G. Pachpatte. On some new inequalities retarde to a certian inequality arising in the theory of differential equations. J. Mat. Anal. Appl., 2000,251 (2000):736-751.

[10] A. A. El-Deeb and Reda Gamal Ahmed. on some genralizations of certain nonlinear retarded integral inequalities for Aolterra-Fredholm integral equations and their applicatins in delay differential equations. J. Eqy. Math. Sco., 25(2017):279-285.

[11] H. El-Owaidy, A. A. Ragab, Wabuelela, and A. A. El-Deeb. on some new nonlinear integral inequalities of GronwallBellman type. kyungpook math, 54 (2014):555-575.

[12] I. A. Bihari. A generalization of lemma of bellman and its application to uniqueness problem of differential equation. Acta.Math.Acad.Sci.Hung, 7:(1956) 81-94.

[13] R. Bellman. The stability of solutions of linear differential equations. Duke Math. J, 10 (1943):643-647.

[14] R. Bellman. A symptotic series for the solutions of linear differential-difference equations. Rendiconti del circolo Matematica Di Palermo, 7(1958):1-9.

[15] H. E. Gollwitzer. A note on a functional inequality. Proc. Amer. Math. Soc., 23(1969):642-647.

[16] B. G. Pachpatte. Inequalities for Differential and Integral Equation. 1998.

[17] B. G. Pachpatte. Integral and Finit Difference Inequalities and Applications. 2006.

[18] S. D. Kender, S. G. Latpate, and S. S. Ranmal. Some nonlinear integral inequalies for volterra-fredholm integral equations. Appl. Adv. Inequal, 21(2014) 11 pages.

[19] S. Hilger. Analysis on measure chains unified approach to continuous and discrete calculus. Results Math, 18 (1990):1856.

[20] M. Bohner and A. Peterson. Advances in Dynamic Equations on Time Scales. 2003.

[21] M. Bohner, L. Erbe, and A. Peterson. Oscillation for nonlinear second order dynamic equations on a time scale. J. Math. Anal. Appl., 301 (2005):491-507. 
[22] V. Kac and P. Cheung. Quantum Calculus. Springer, New York, 2001.

[23] M. Bohner and A. Peterson. Dynamic Equations on Time Scales. 2001.

[24] W. N. Li and M. Han. Bounds for certain nonlinear dynamic inequalities on time scales. Discrete Dyn. Nat. Soc, 2009 (2009):14 pages.

[25] W.N. Li, Q. Zhang, and F. Qiu. Some nonlinear delay discrete inequalities and their applications. Demonstratio Math, 36(2006):771-782.

[26] R.P. Agarwal, M. Bohner, and A. Peterson. Inequalities on time scales. A survey, Math. Inequal. Appl., 4 (2001):535-557.

[27] W. N. Li. Some pachpatte type inequalities on time scales. Comp. Math. Appl, 57 (2009):275-282.

[28] D. S. Mitrinovic. Analytic Inequalities. Springer-Verlag, Berlin/New York, 1970. 